\title{
Design and Testing of a Compliant Mechanism-Based Demonstrator for a Droop-Nose Morphing Device
}

\author{
Srinivas Vasista ${ }^{1}$ Johannes Riemenschneider $^{2}$ and Hans Peter Monner ${ }^{3}$ \\ German Aerospace Center (DLR), Braunschweig, 38108, Germany
}

\begin{abstract}
A demonstrator morphing leading edge was designed and manufactured as an intermediary step in preparation for wind tunnel testing of a droop-nose adaptive morphing wingtip (AMWT) as part of the European FP7 project NOVEMOR. This demonstrator features a flexible fiberglass skin and a monolithic aluminum internal compliant mechanism and support structure for lightweight design. The design process involves the design of the skin via a structural optimization tool, followed by continuum gradient-based topology optimization of first the compliant mechanism and then the support structure. The skin was manufactured using prepreg Hexcel HexPly ${ }^{\circledR} 913$ plies, the aluminum internal structure was laser cut from stock plate material and the compliant region was driven by a linear stepper motor actuator. Displacements and strains were measured and compared with target values and that of finite element computations and overall show good agreement; however issues such as grey-areas and hinge-regions in the topology optimization need to be addressed for the final wind tunnel design for better post-processing and reduced stress concentrations.
\end{abstract}

\section{Nomenclature}

area of finite element

$=$ mean compliance

$=$ coefficient of lift

$=$ finite element analysis force vector

$=$ shape control constraint function

$=$ displacement error function

$=$ current element in mesh

$=$ shape control objective function

$=$ finite element analysis stiffness matrix

$=$ Mach number

$=$ stiffness design constraint function

$=$ number of elements in mesh

$=$ number of control degrees of freedom

$=$ current control degree of freedom

$=$ current load case

$=$ finite element analysis displacement vector

$=$ stiffness design volume fraction

$=$ shape control design volume fraction

$=$ topology optimization density design variable

$=$ shape control topology optimization actuation force magnitude design variable

\footnotetext{
1 Alexander von Humboldt Postdoctoral Research Fellow, Department of Adaptronics, Institute of Composite Structures and Adaptive Systems, Lilienthalplatz 7, Braunschweig 38108, Germany. AIAA member

${ }^{2}$ Deputy Head of Department, Department of Adaptronics, Institute of Composite Structures and Adaptive Systems, Lilienthalplatz 7, Braunschweig 38108, Germany.

3 Head of Department, Department of Adaptronics, Institute of Composite Structures and Adaptive Systems, Lilienthalplatz 7, Braunschweig 38108, Germany. Senior AIAA Member
}

American Institute of Aeronautics and Astronautics 


\section{Introduction}

T has been identified under the European 7th Framework Programme (FP7) that there is need for development of integrated, safer, greener and smarter pan-European sustainable transport systems with specific emphasis on the reduction of emissions and environmentally efficient aviation ${ }^{1}$. The implementation of morphing structures in aircraft has the potential to address these challenges and reduce pollution and noise emissions, and research in this field is growing in significance in light of such potential advantages ${ }^{2,3}$. Among the many applications of morphing structures is the use of smoothly morphing gapless control surfaces and high-lift devices as enablers for airflow laminarization, a technique from which significant drag reductions may be achieved within a suitable timeframe ${ }^{4}$.

This paper presents research conducted in the European FP7 project NOVEMOR, in which an adaptive morphing wingtip (AMWT) of the form of a leading-edge droop-nose morphing device is currently under design. The leading edge of the AMWT consists of a flexible composite skin with a tailored thickness distribution which droops smoothly based on input forces from actuators transmitted through internal mechanisms. The reference aircraft is a regional jetliner with geometry and aerodynamic data provided by project partner Embraer, as shown in Fig. 1, at two different configurations (clean: $M 0.78, C_{L} 0.47$ and droop: $M 0.3, C_{L} 0.6$ ). The AMWT device was envisaged as a suitable candidate for drag reduction, as well as to overcome some aeroelastic problems that classical wing control surfaces can encounter such as aileron efficiency loss with increasing dynamic pressure. Furthermore, the wing lift distribution may be adjusted in flight by the AMWT to best suit the prevailing conditions, resulting in higher overall efficiency. One key feature of the AMWT is the use of compliant mechanisms to transfer the actuation force to the skin instead of conventional rigid-link mechanisms, with the anticipated benefits including potential weight savings, reduced part and assembly costs, and the elimination of backlash. A full scale experimental model is scheduled to be wind tunnel tested in February 2015 as an evaluation of structural behavior under aerodynamic loads.
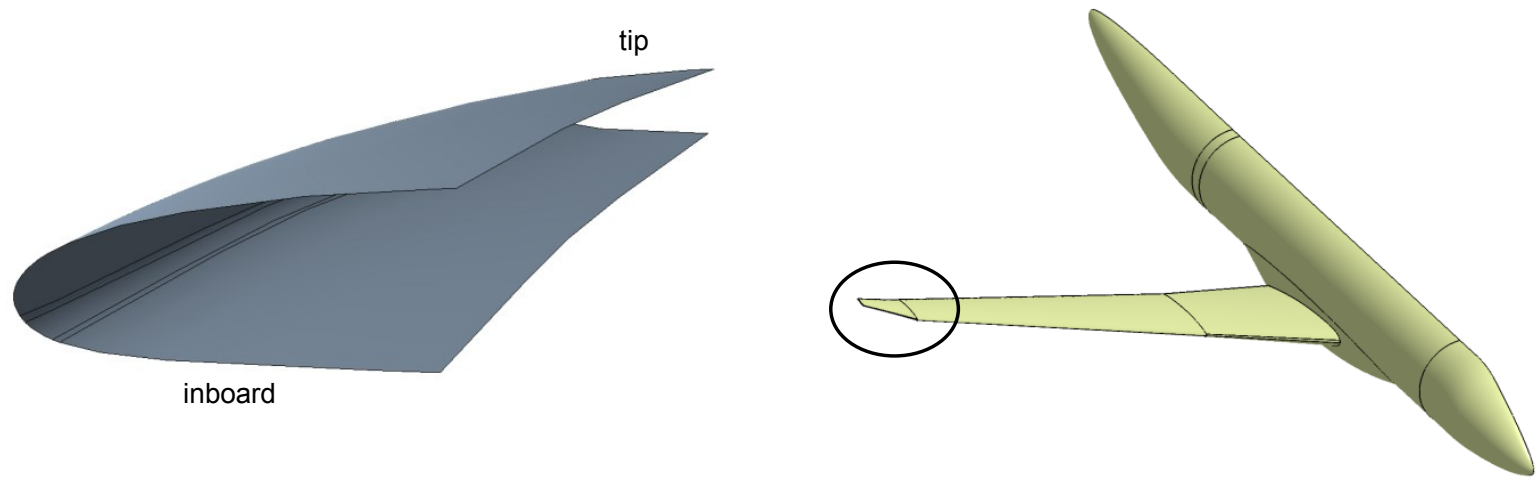

Figure 1. 3D geometry of aircraft and wingtip leading edge.

The focus of this paper is the structural design procedure and experimental ground testing of a demonstrator model for the AMWT, constructed as an intermediary step to acquire knowledge for the manufacture and testing of the final wind tunnel model. Of particular interest is the assessment of the performance of the compliant mechanism. The design chain and the numerical tools developed and applied in this process are first outlined and the postprocessing and manufacturing procedures are subsequently described. Results from ground testing are then presented and the conclusions drawn are stated.

\section{Structural Design Process}

One of the key requirements of a morphing wing device, in general, is that the outer profile of the structure should be able to assume multiple shapes under different actuation states and external (e.g. aerodynamic) loads with minimal deviation from the specified target profiles. This applies to the AMWT device where the leading edge profile of the wingtip needs to conform to the clean target shape without actuation input and the droop shape when actuated. The 3D clean and droop geometry was defined by 2D profiles at five stations along the span, shown in Fig. 2 , and features an approximate droop angle of $2^{\circ}$. 

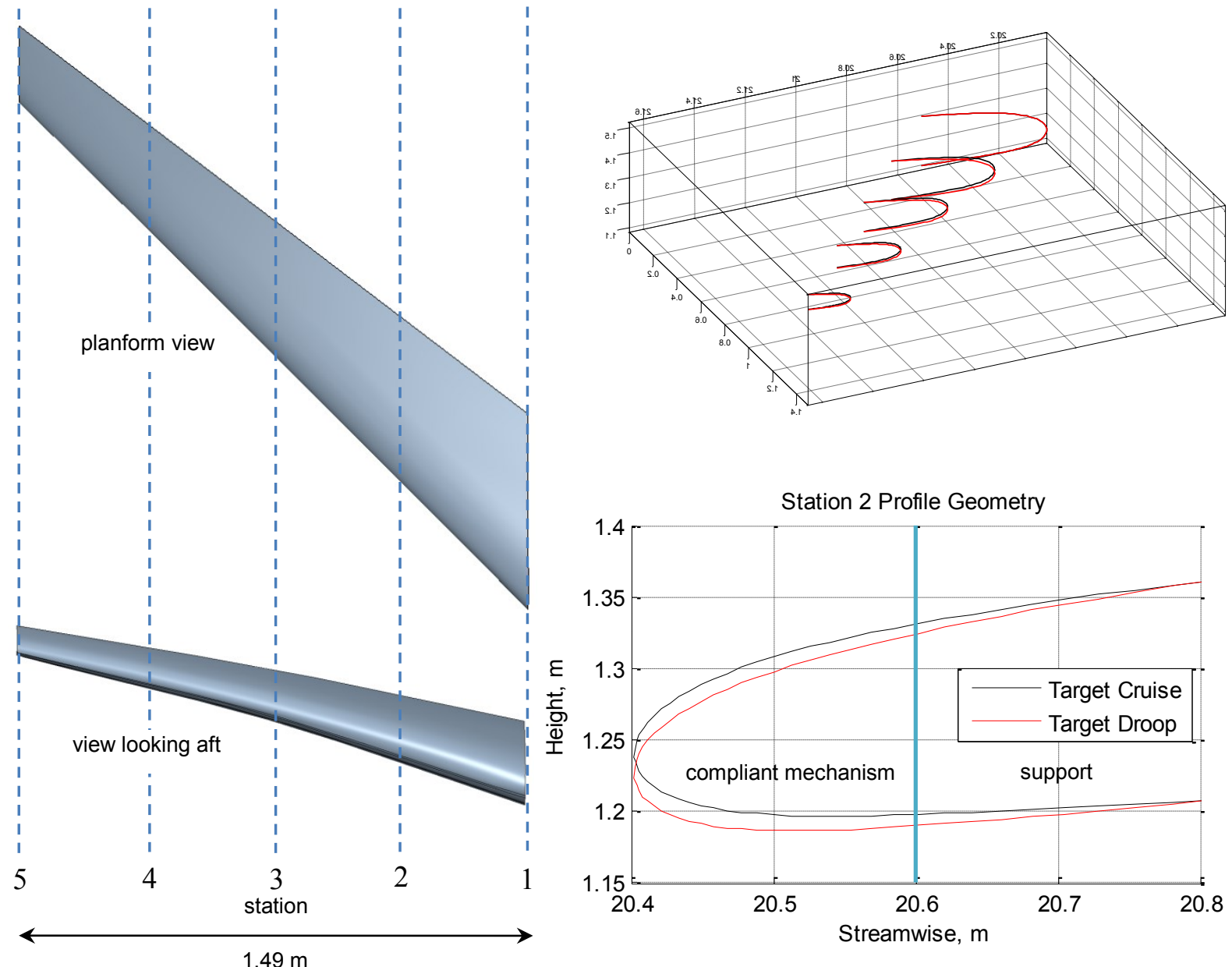

Figure 2. Geometry of the wingtip leading edge showing sweep, dihedral, aerofoil target cruise and droop for the five stations and detailed view for station 2 .

The overall structural concept comprises a fiberglass composite material skin with an optimized thickness distribution and a double-L stringer placed at an optimal position. The reader is referred to Ref. 5 for further information on the 3D skin design aspects. The stringer provides a connection point to the mechanism and thus is the location where force from the mechanism is introduced onto the skin. The stringer also provides additional bending stiffness in the spanwise direction. The demonstrator design features a span length of $100 \mathrm{~mm}$ with a uniform profile based on the $2 \mathrm{D}$ geometry of station 2 . This profile geometry was divided into two segments: the forward segment being the region of the compliant mechanism and aft region being the support which connects the compliant mechanism to the fixed spar. One planar compliant mechanism was used, driven by one linear actuator acting in the horizontal chordwise direction. The compliant mechanism and support were considered as being manufactured from a single piece of material to simplify the build process and for a lightweight design. In such a configuration, this compliant mechanism/support component takes the form of a compliant leading edge rib.

The basis of the structural design process follows that of Refs. 4 and 6 and for this work consists of three optimization stages as shown in Fig. 3: 1) the design of the skin via Simplex ${ }^{7}$ based optimization; 2) the design of the compliant mechanism via continuum gradient-based topology optimization and 3) the design of the support for the compliant mechanism also via continuum gradient-based topology optimization. The remainder of this section details each stage. 
- Geometry (clean and droop profiles)

- Skin material properties

- Aerodynamic loads

- Stringer type
- Mechanism material properties

- Thickness

- Actuator type, properties - Volume fraction and location

- Fixed support location
- Fixed support location

- Actuator support

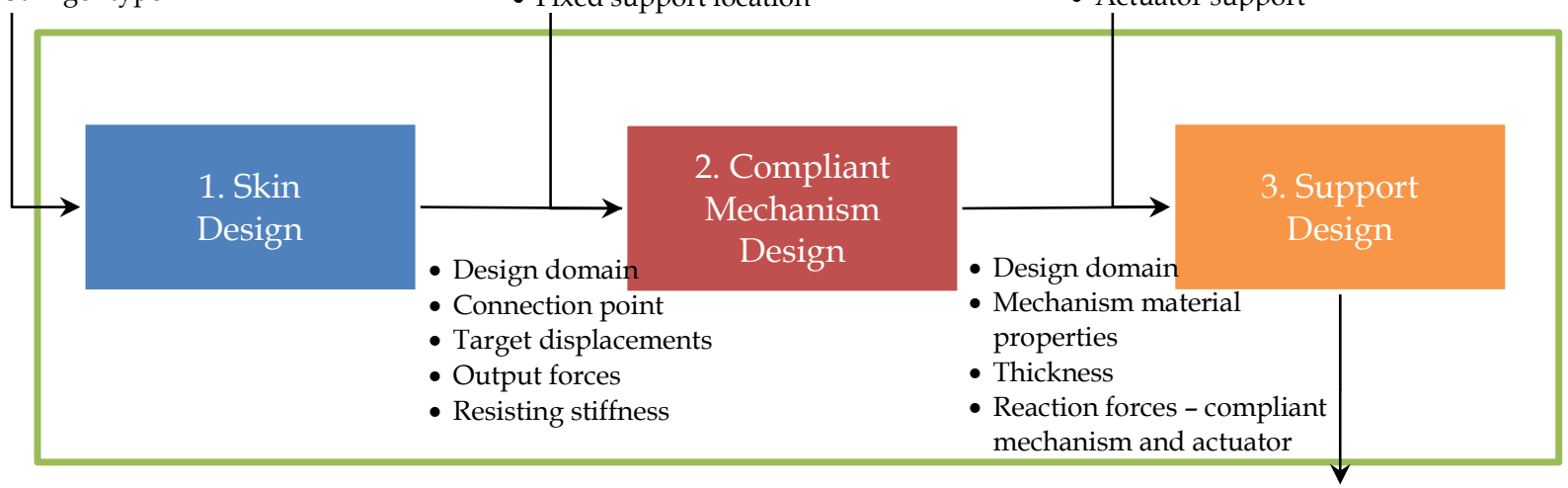

AMWT

Figure 3. Schematic of the design process of the adaptive morphing wingtip.

\section{A. Skin Design}

1. Skin Optimization Setup

The DLR design tool ${ }^{4}$ was used for the design of the skin and features a Simplex search optimization method. For the design of the demonstrator model, a uniform skin thickness of $2.75 \mathrm{~mm}$ was assumed due to the relatively small droop target displacement and for the purpose of reducing manufacturing complexity. The specified thickness is achieved according to a stacking sequence based on HexPly ${ }^{\circledR} 913$ prepreg plies. The design variables were the position of the stringer along the perimeter of the profile and the magnitude of the displacement required from the mechanism at the attachment points at the stringer. The process requires a number of finite element analysis calculations for each optimization iteration, generating displacement and strain solutions for the cruise and droop configurations. The finite element modelling consists of $3 \mathrm{D}$ shell elements comprising the skin and the stringer for a span length of $100 \mathrm{~mm}$.

\section{Skin Optimization Results}

The resultant deformation profiles of the skin are shown in Fig. 4 along with the position of the stringer and the maximum strain. These results show a good agreement between the target and actual shapes and with skin strain values well below the material limits. Information from the skin model is required as input for the compliant mechanism design once the skin design process is completed. These include the design domain geometry, location of the connection points, target displacements and output forces to be delivered by the mechanism, and the stiffness of the skin, which was exported as effective system stiffnesses at the control points and performed using a substructuring approach. The values of the relevant data transferred from the skin model are shown in Table 1. 




Figure 4. Results of the skin optimization process.

\section{B. Compliant Mechanism Design}

\section{Shape Control Topology Optimization Problem Formulation}

The function of the compliant mechanism is to transmit the force and stroke from the actuator when actuated onto the skin (via the stringer) such that the skin conforms to the droop shape with minimal error, and also to support the skin against deformation in the clean configuration. The mechanism needs to work against the aerodynamic loads and the stiffness of the skin and these boundary conditions need to be considered in the modeling. The premise behind the compliant mechanism design is that the skin should conform to the target profile if the skin-mechanism connection points move to their target positions, thereby forming a shape control problem. Topology optimization methods have been used for shape control problems and can be loosely classified in three categories: i) use of the discrete load path representation and optimization update schemes such as genetic algorithms ${ }^{8,9,12}$; ii) the use of continuum gradient-based topology optimization methods for the bending shape control of plates $^{10,11}$; and iii) the use of continuum gradient-based topology optimization methods for the design of planar morphing structures ${ }^{12-15}$. This work falls into the last category and efforts are made in this work to develop the topology optimization tool to a level where, starting from essentially a "blank canvas", a functional structure can be physically realized, implying that interpretation and post-processing of the final topology are feasible.

The objective function was to minimize the least squares error between the target and actuated displacements of the control points, subject to constraints on the amount of material used and the finite element governing equations as shown in Eq. 1. The design variables were the topological densities $\mathbf{x}$, and the magnitude of the actuation force $\alpha$. The solid isotropic material with penalization (SIMP) material model ${ }^{16}$ was used in conjunction with sensitivity analysis and the method of moving updates ${ }^{17}$ (MMA) as the design update scheme. The implementation of this type of least squares problem with MMA was performed as suggested in Ref. 18, whereby the shape control objective function was essentially implemented as displacement constraints. The sensitivities of the displacement and material constraints to changes in design variables were calculated using the adjoint method. The design domain is shown in Fig. 5 along with the finite element mesh and boundary conditions and other relevant design parameters including the target displacements are shown in Table 1. The material selected for the compliant mechanism was aluminum 7075 alloy due to the relatively high value of the effective yield strain (approximately $0.7 \%$ ) with a thickness of 5 $\mathrm{mm}$ to ensure sufficient stiffness and also to ensure sufficient width for strain gauge placement for the experimental stage. A linear actuator was also considered due to the slender nature of the profile geometry and space restrictions. 


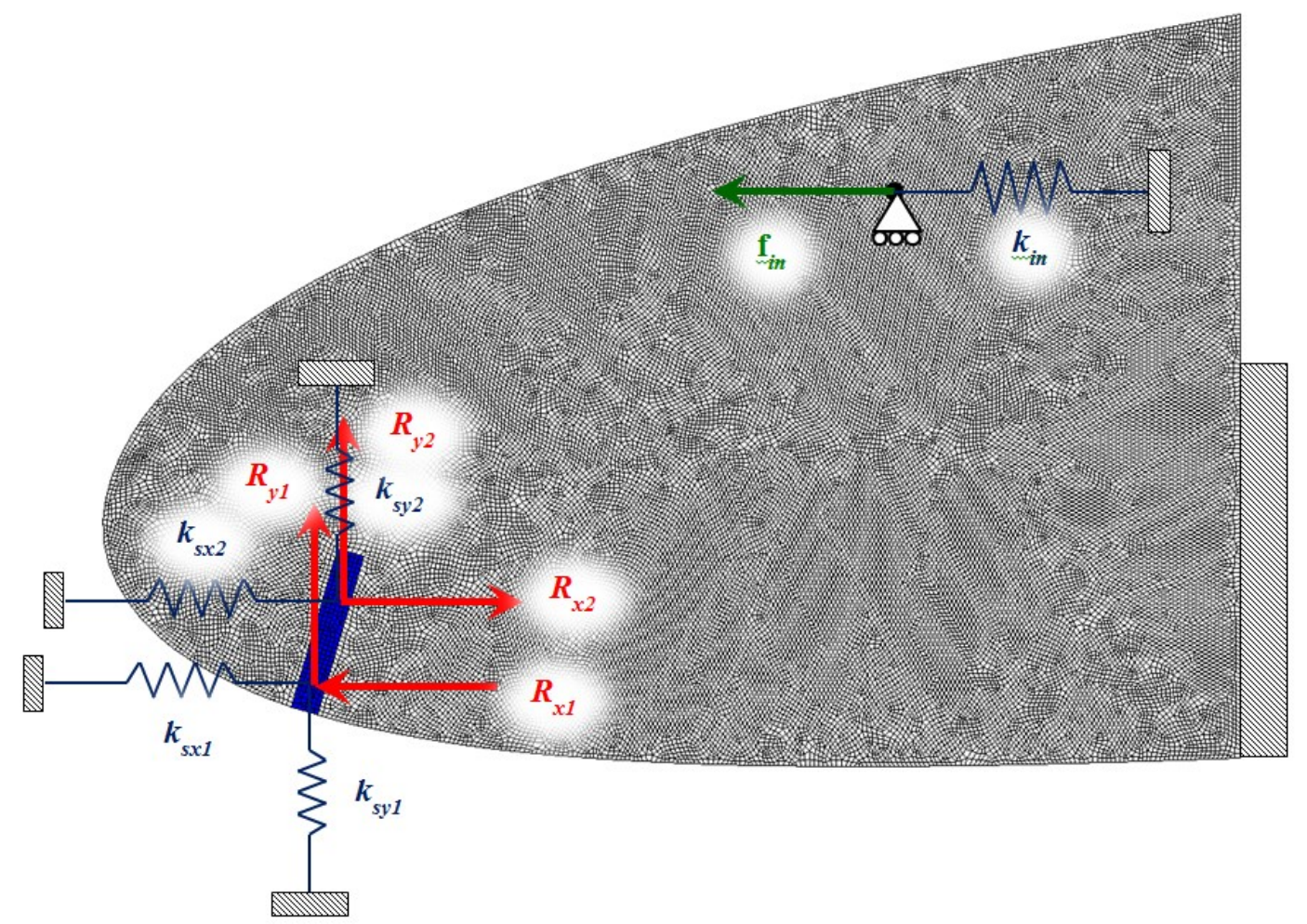

Figure 5. Design domain, mesh, boundary conditions and loads for topology optimization of the compliant mechanism.

Table 1. Parameters transferred from the skin optimization stage to the topology optimization process.

\begin{tabular}{cc}
\hline \hline Parameter & Value \\
\hline \hline$k_{s x l}$ & $278.50 \mathrm{~N} / \mathrm{mm}$ \\
$k_{s y l}$ & $2.09 \mathrm{~N} / \mathrm{mm}$ \\
$k_{s x 2}$ & $37.65 \mathrm{~N} / \mathrm{mm}$ \\
$k_{s y 2}$ & $2.08 \mathrm{~N} / \mathrm{mm}$ \\
$R_{x l}$ & $-5.35 \mathrm{~N}$ \\
$R_{y 1}$ & $0.54 \mathrm{~N}$ \\
$R_{x 2}$ & $18.99 \mathrm{~N}$ \\
$R_{y 2}$ & $-9.48 \mathrm{~N}$ \\
$f_{i n}$ & $340 \mathrm{~N}(\mathrm{max})$ \\
$k_{\text {in }}$ & $30.00 \mathrm{~N} / \mathrm{mm}$ \\
$u_{x-\text { tarl }}$ & $1.22 \mathrm{~mm}$ \\
$u_{y \text {-tarl }}$ & $-12.02 \mathrm{~mm}$ \\
$u_{x-\operatorname{tar} 2}$ & $1.47 \mathrm{~mm}$ \\
$u_{y-\text { tar }}$ & $-12.10 \mathrm{~mm}$ \\
\hline
\end{tabular}




$$
\begin{gathered}
\min _{\mathbf{x}, \alpha}: J(\mathbf{x}, \alpha)=\sum_{r=1}^{p}\left(h_{r}(\mathbf{x}, \alpha)\right)^{2} \\
\text { s.t. }: g(\mathbf{x})=\frac{\sum_{i=1}^{N} x_{i} A_{i}}{w \sum_{i=1}^{N} A_{i}}-1 \leq 0 \\
\quad \mathbf{K u}=\mathbf{f} \\
\mathbf{0} \leq \mathbf{x} \leq \mathbf{1} \\
0<\alpha \leq 1
\end{gathered}
$$

where

$$
h_{r}(\mathbf{x}, \alpha)=u_{r}(\mathbf{x}, \alpha)-\bar{u}_{r}
$$

\section{Compliant Mechanism Topology Optimization Results}

The results of the compliant mechanism topology optimization process are shown in Fig. 6. The topology features several linear members and a number of hinge-like regions. The plot of the actuated mechanism shows that the mechanism can move the control points to the target location appropriately and this is supported by the plot of the objective function history showing clear minimization of the displacement error. Data from the compliant mechanism is required for the design of the support. These are the design domain geometry, material and thickness (as the design is monolithic), reaction forces from the compliant mechanism and the actuator and their locations. Further design considerations include the required volume fraction, the location of the fixed support, and the actuator mount attached to the support. It should be noted that the actuator mount location is determined by consideration of the assembly of the demonstrator and this is described in Section III-A.

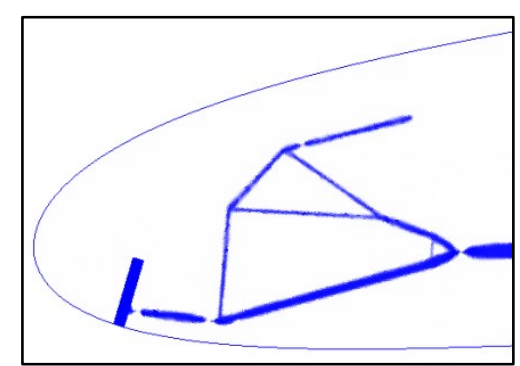

a)

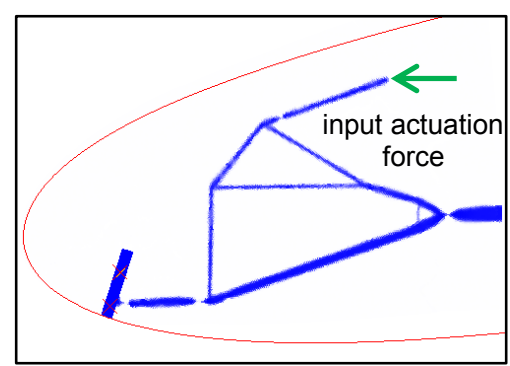

b)

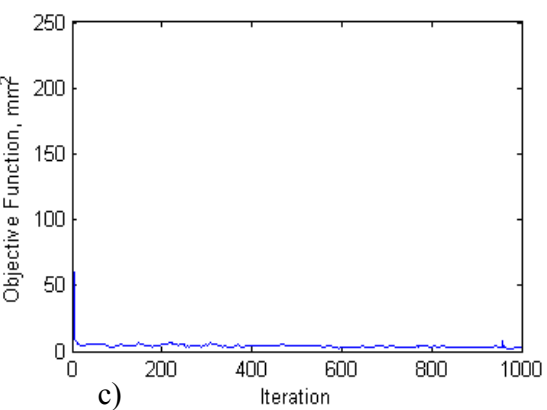

c)

Figure 6. Resultant penalized topologies of the compliant mechanism in a) the unactuated state and b) the actuated state; objective function (squared displacement error sum) history.

\section{Support Design}

1. Support Design Topology Optimization Formulation

The support was designed using topology optimization in which a minimum mean compliance problem was solved. The design domain, boundary conditions and loads from the compliant mechanism and actuator mount are shown in Fig. 7. The loads were extracted at the boundaries of the interface between mechanism and support as well as extrapolated from actuator location onto the shaft support point. The order of the loads is $388 \mathrm{~N}$ in the $\mathrm{x}$-direction and $125 \mathrm{~N}$ in the y-direction. In order to ensure robustness of the support at off-design conditions, two load cases were considered: 1) the loads determined directly from the compliant mechanism process and 2) complete reversal of these loads as an extreme design case. The problem statement is given in Eq. 2 where the objective was to minimize the mean compliance from the two load cases subject to a constraint on the maximum allowable volume fraction (0.3). The design variables were the topological densities and adjoint-based sensitivity analysis was used in conjunction with the MMA routine. 




compliant mechanism reaction forces

Figure 7. Design domain, mesh, boundary conditions and loads for topology optimization of the support.

$$
\begin{aligned}
\min _{\mathbf{x}, \alpha}: C & =\sum_{t=1}^{2} \sum_{i} \mathbf{u}_{i_{t}}{ }^{T} \mathbf{k}_{i} \mathbf{u}_{i_{t}} \\
\text { s.t. : } m & =\frac{\sum_{i=1}^{N} x_{i} A_{i}}{v \sum_{i=1}^{N} A_{i}}-1 \leq 0 \\
& \mathbf{K u}=\mathbf{f} \\
\mathbf{0} & \leq \mathbf{x} \leq \mathbf{1}
\end{aligned}
$$

\section{Support Design Topology Optimization Results}

The results of the support design topology optimization are shown in Fig. 8. The support structure comprises truss and brace features, resulting in a stiff structure as further validated by the objective function history showing minimization of the mean compliance.
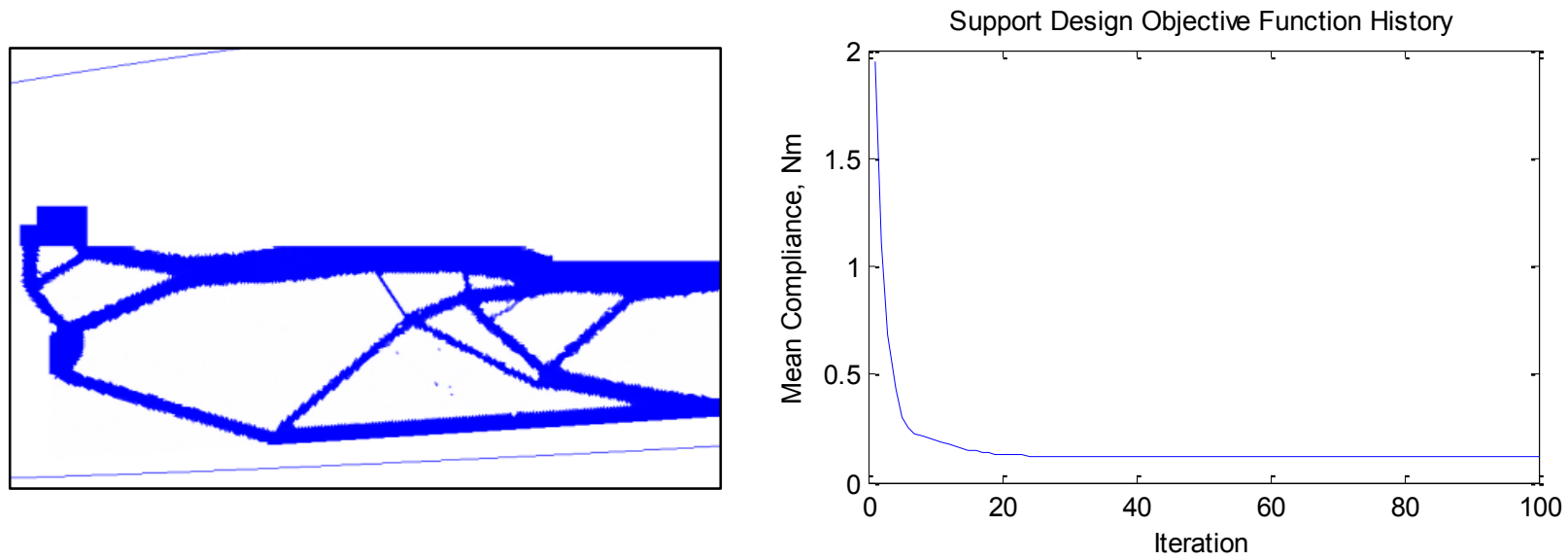

Figure 8. Resultant topology and objective function history of the support design process. 


\section{Manufacturing of Demonstrator}

\section{A. Demonstrator Concept}

The CAD concept and manufactured demonstrator are shown in Fig. 9. A rear spar acts as a mount for the skin, internal monolithic compliant mechanism/support and linear stepper motor. The stringer is attached to the compliant mechanism via bolts and reinforcing steel strips in order to distribute the force from the mechanism over a greater spanwise distance of the stringer. Actuating the motor results in a linear motion of the shaft which drives the compliant mechanism and droops the skin.

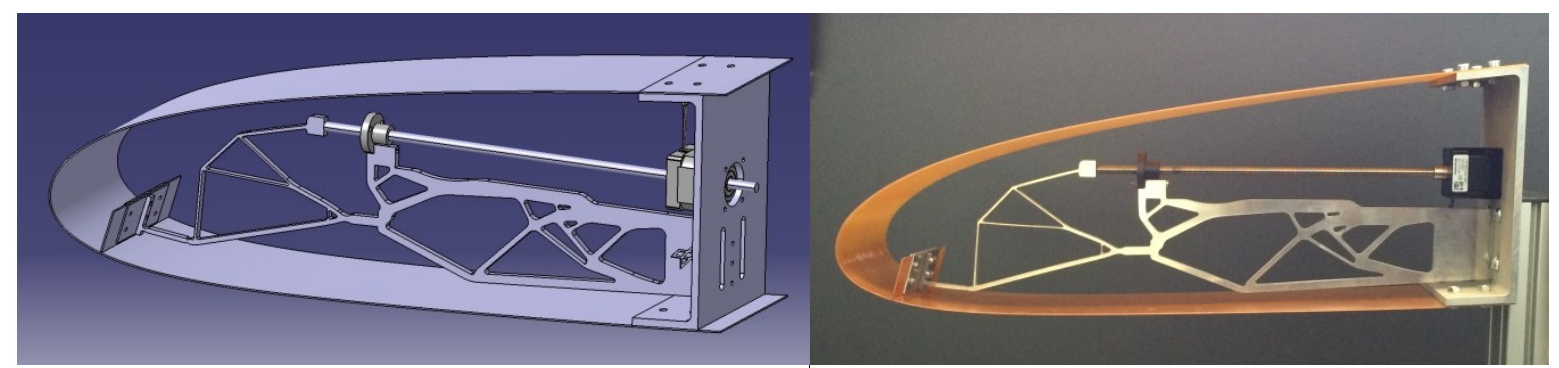

Figure 9. CAD conceptual model and manufactured model of the demonstrator.

\section{B. Skin}

Two skins with thicknesses of 1 and $2 \mathrm{~mm}$ were fabricated to test the effect of different stiffnesses that the compliant mechanism needs to act against. The design thickness $(2.75 \mathrm{~mm})$ of the skin was not manufactured as initial finite element analysis results showed that the actuator would exceed its maximum recommended force limit with the added thickness. Both skins were manufactured by using $0.125 \mathrm{~mm}$ thick Hexcel HexPly ${ }^{\circledR} 913$ prepreg plies, with symmetric sequences of $0 \% 0^{\circ} /-45^{\circ} /+45^{\circ} / \mathrm{sym}$ and $0 \% 0^{\circ} /-45^{\circ} /+45^{\circ} / 0^{\circ} /-45^{\circ} / 90^{\circ} /+45^{\circ} / \mathrm{sym}$ for the $1 \mathrm{~mm}$ and $2 \mathrm{~mm}$ thick skins respectively. The aluminum mold is shown in Fig. 10 with the position of the stringer and cut ends marked. The stringer was constructed integrally by 'pulling-up' four layers from the forward and aft sections, resulting in a thickness of $1 \mathrm{~mm}$ for both skins.



Figure 10. Aluminum mold showing stringer position and cut ends for the manufacture of the skin.

\section{Internal Mechanism and Support Structure}

\section{Topology Post-processing}

A topology post-processing stage was required to convert the topological densities into a real part with a defined geometry. Some difficulties exist in interpreting the contour of the compliant mechanism due to the presence of hinge-like regions and some boundary elements having an intermediate-valued topological density and are currently being addressed for the final wind tunnel model. The topology of the compliant mechanism was interpreted as comprising several linear members connected by hinge-like regions. A parametric model was set up in which the thicknesses of the linear members, and the thickness and length of the hinge-like features were variables. An automated procedure linking the CAD geometry to the finite element solver was set up and the displacement and 
maximum stress results assessed to determine the best values of these parametric variables. This post-processing procedure was not required for the support, as the topology was well defined and without hinge-like regions.

\section{Fabrication}

The internal monolithic compliant mechanism/support was laser cut from a $5 \mathrm{~mm}$ thick aluminum 7075 alloy plate. Mounting holes to the spar, actuator and skin were subsequently drilled.

\section{Actuation System}

A non-captive linear actuator stepper motor was used to drive the compliant mechanism. The internal shaft was supported by a nut mounted to the internal support structure to reduce the bending moment on the shaft and to prevent any shaft play. Anti-rotation of the shaft was achieved by inserting the shaft end into the compliant mechanism and clamping the shaft with bolts within the compliant mechanism.

\section{Testing and Results}

\section{A. Material Testing}

As mentioned afore, aluminum 7075 was selected as the material for the monolithic compliant mechanism and support due to its relatively high ratio of yield strain to elastic modulus as reported in various material handbooks. Material testing in the form of tensile tests was conducted in order to ascertain the modulus of elasticity and stress and strain yield and ultimate limits to accurately determine the working limits of the specimen used in the demonstrator and also to ensure that the topology optimization and finite element modelling calculations were valid. Four tensile test specimens were cut from the same aluminum plate (extruded) used for the compliant mechanism, with two specimens aligned in the $\mathrm{x}$-axis of the compliant mechanism (H1 and H2) and the other two orthogonally in the y-direction (V1 and V2). Strain gauges were applied on test specimens for strain measurement and the displacement and force data were obtained from the tensile test machine itself. The resulting stress-strain curve is shown in Fig. 11 and the average elastic modulus and ultimate stress limit measured to be $69.7 \mathrm{GPa}$ and $524 \mathrm{MPa}$ respectively, with approximately $2.25 \%$ variation over the four specimens. Overlaid in the stress-strain plot is the strain value at yield stress which is 0.0075 , corresponding closely with values reported in handbooks. These values also indicate that the material values used in the topology optimization and finite element modelling computations are valid.

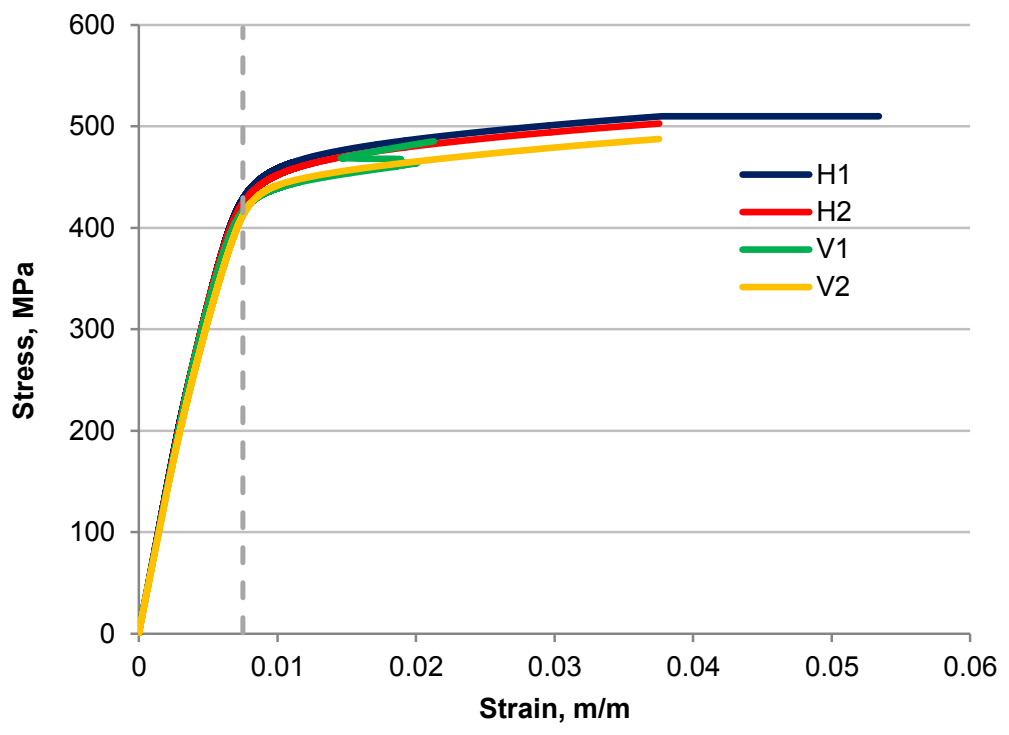

Figure 11. Aluminum 7075 experimentally measured stress-strain curves. 


\section{B. Compliant Mechanism Displacements}

The overall shape of the leading edge in clean and droop configurations for the $1 \mathrm{~mm}$ skin is shown in the montage in Fig. 12a. The displaced solution from the finite element results also shows that the overall desired profile has been achieved. The displacements at the compliant mechanism output points (i.e. attachment region to stringer) were measured using image analysis techniques and compared with target and finite element results. A 14.2 megapixel SLR camera was mounted and parallel with the compliant mechanism axes and fixed securely in close proximity to the measuring points to minimize parallax errors. The $\mathrm{x}$ - and $\mathrm{y}$ - displacements at the top and bottom measuring points as shown in Fig. 12b were measured by means of pixel distance and subsequently post-processed in an image analysis software. Figure 13 shows the finite element solutions for both skins with von Mises contours overlaid.

The results are shown for both the $1 \mathrm{~mm}$ and $2 \mathrm{~mm}$ skin thicknesses in Fig. 14a and 14b and it should be noted that the input displacement of the actuator was constant at $5 \mathrm{~mm}$. The finite element result and measured values shows good agreement for the $1 \mathrm{~mm}$ thick skin and slightly more deviation for the $2 \mathrm{~mm}$ thick skin. However, when compared with the target displacements it is clear that the mechanism has less displacement in the y-direction and more in the $\mathrm{x}$-direction than is necessary for both the finite element and measured cases. This indicates that the displacement is more of a rotation about the hinge-like region. This discrepancy can be due to the grey elements in the topology result and the hinge-like regions which are difficult to interpret and post-process. Considerations to overcome these limitations are being investigated for the compliant mechanisms in the final wind tunnel model. Comparing the $1 \mathrm{~mm}$ thick skin to the $2 \mathrm{~mm}$ thick skin, the finite element results show a small reduction in output displacement, in the order of $0.5 \mathrm{~mm}$ in the y-direction. However, the experimental results show a larger reduction of the output displacement in the order of $1.2 \mathrm{~mm}$ in the y-direction. These discrepancies may be possibly due to small local deformation in regions near the stringer and/or actuator connection bolts.

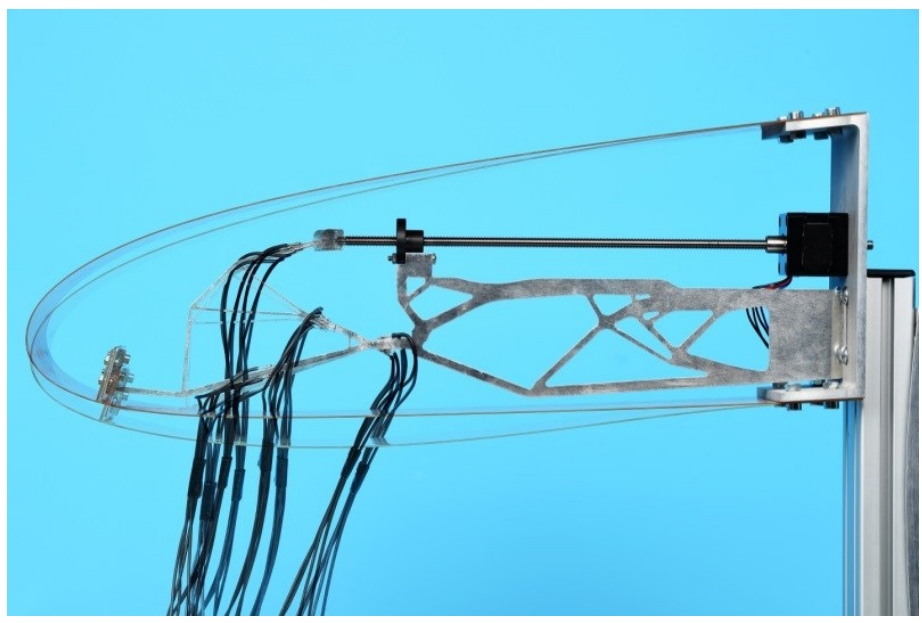

a)

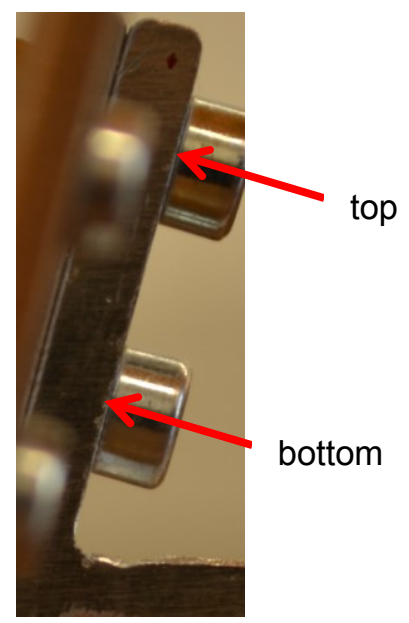

b)

Figure 12. a) Montage of demonstrator in clean and droop configurations; b) measuring points of displacement as used in the image analysis process. 


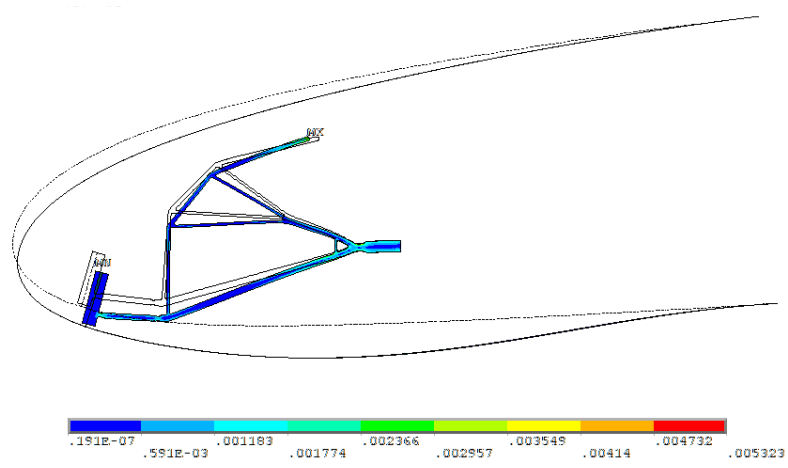

a)

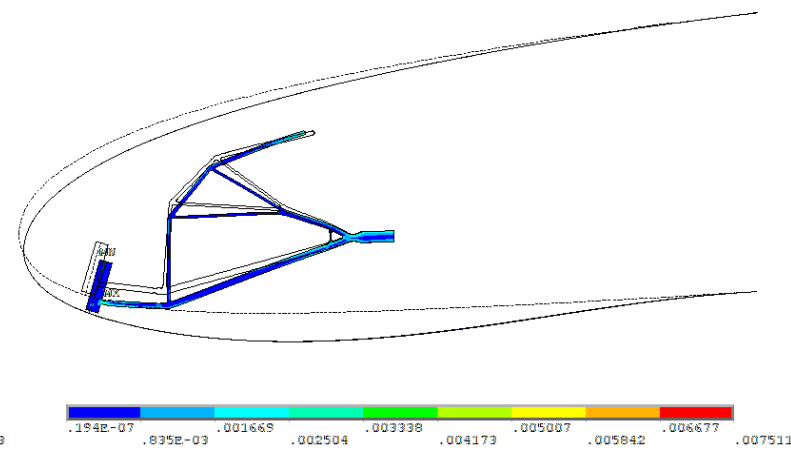

b)

Figure 13. Finite element analysis displacement results with von Mises strain contours for a) the 1 mm skin and b) 2 mm skin.

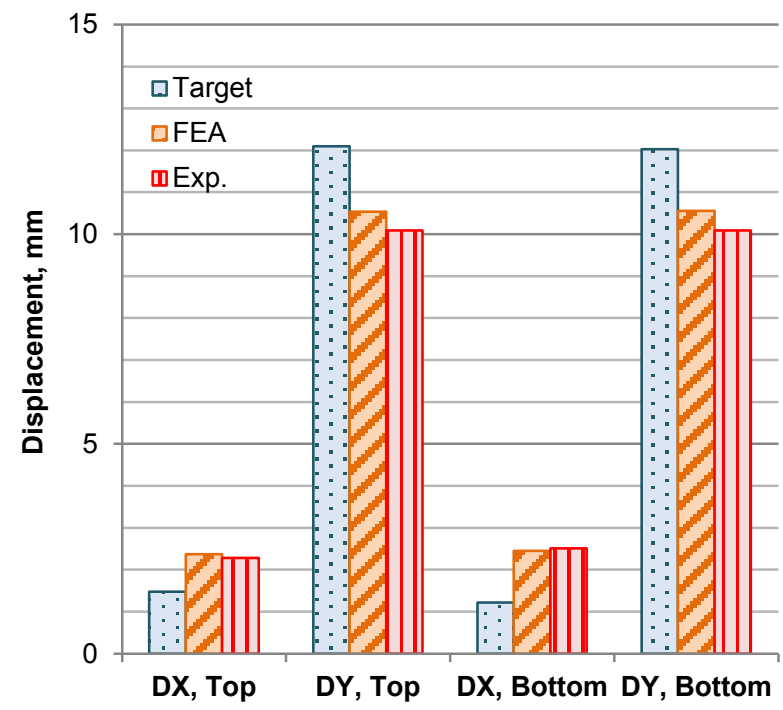

a)

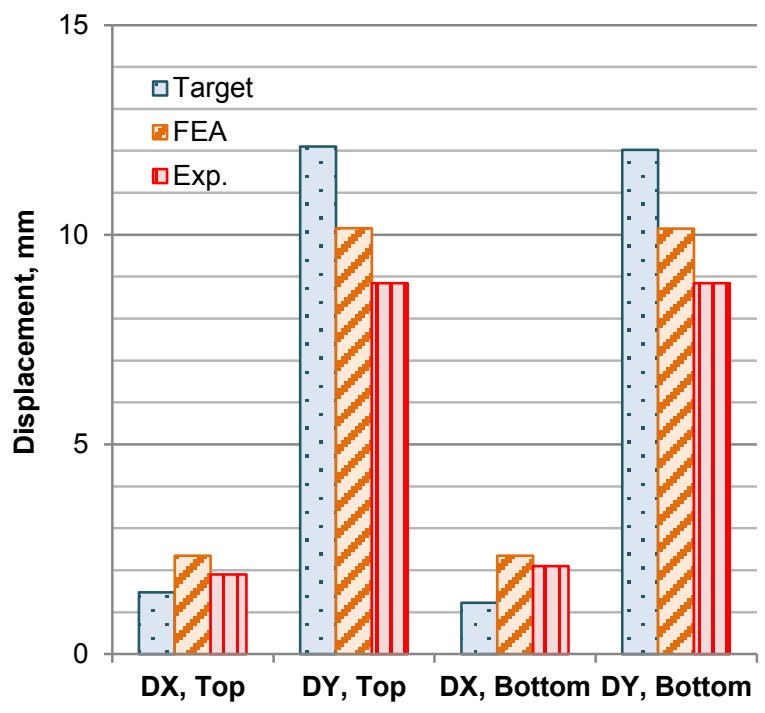

b)

Figure 14. Compliant mechanism displacement results and comparison with target and finite element analysis result for a) the $1 \mathrm{~mm}$ skin and b) $2 \mathrm{~mm}$ skin.

\section{Compliant Mechanism Strains}

Strain gauges were used to measure the strains in various regions of the compliant mechanisms for both the 1 $\mathrm{mm}$ and $2 \mathrm{~mm}$ thick skins. Figure 15 shows the position on the compliant mechanism of the five different regions where strain gauges were placed. It should be noted that positions 1 to 3 were in a half-bridge configuration, thereby effectively measuring the combined bending strain of the upper and lower regions of the corresponding member of the compliant mechanism, while positions 4 and 5 were in quarter-bridge configurations at the hinge-like region thus measuring the strain of the top and bottom surfaces individually.

The results versus time and including input displacement are shown in Fig. 16a and 16b. There is slight nonlinearity of the strains with linearly increasing input displacement for both skins and the strains return to zero when the actuator returns to the stowed position. The results comparing the finite element solution and the experimental values are shown in Fig. 17. There is good agreement between the strains at positions 4 and 5 where the quarter bridge configuration were used, with measured values being approximately 0.45 and $0.4 \%$ for positions 4 and 5 respectively, and under the material strain limit. However, there are some discrepancies in the combined strains, most notably in the position 1 strain of the $2 \mathrm{~mm}$ thick skin and the position 3 strain of the $1 \mathrm{~mm}$ thick skin. These discrepancies may arise from the local deformations near the actuator connection point or may be due to slight 
variations from the applied strain gauge direction to that of the principal strain direction from finite element analysis results.

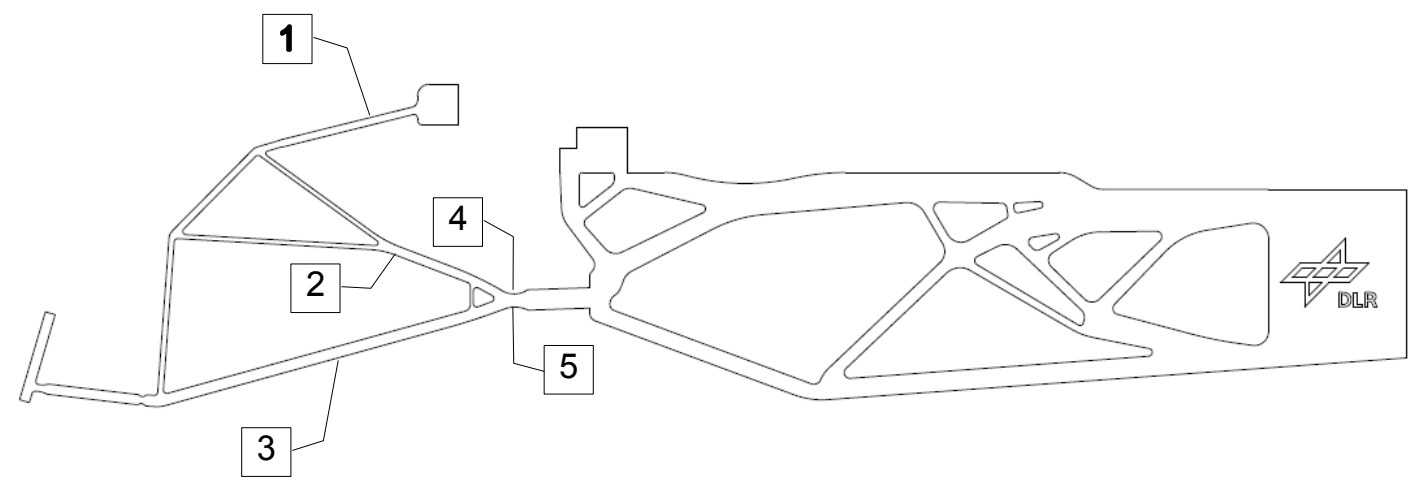

Figure 15. Strain gauge positions on the compliant mechanism.

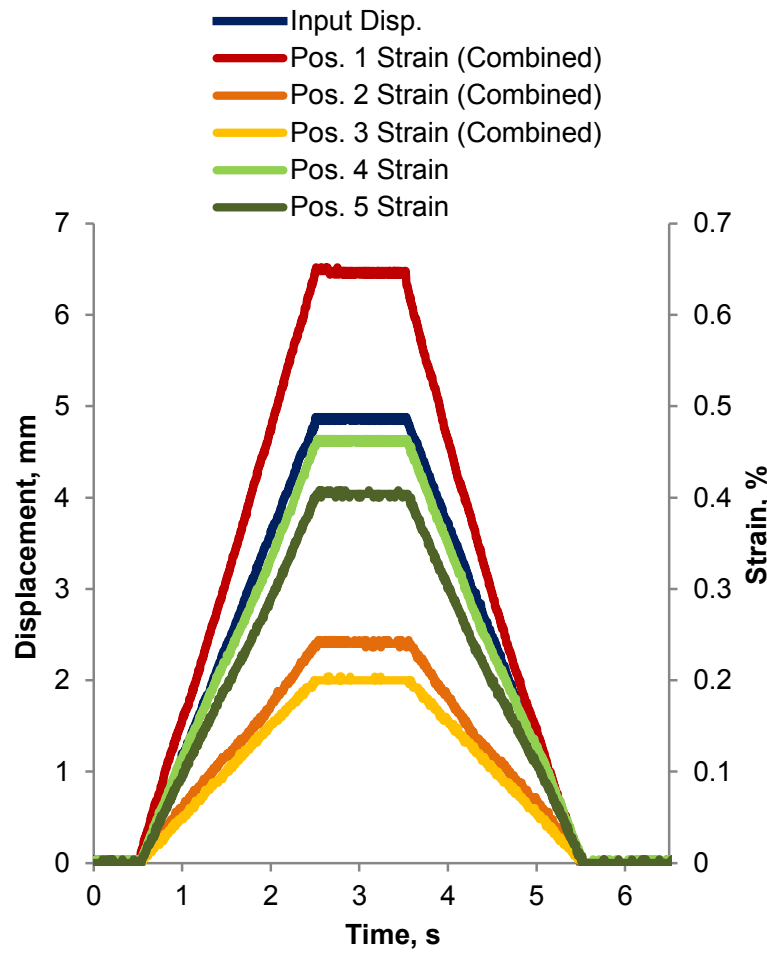

a)

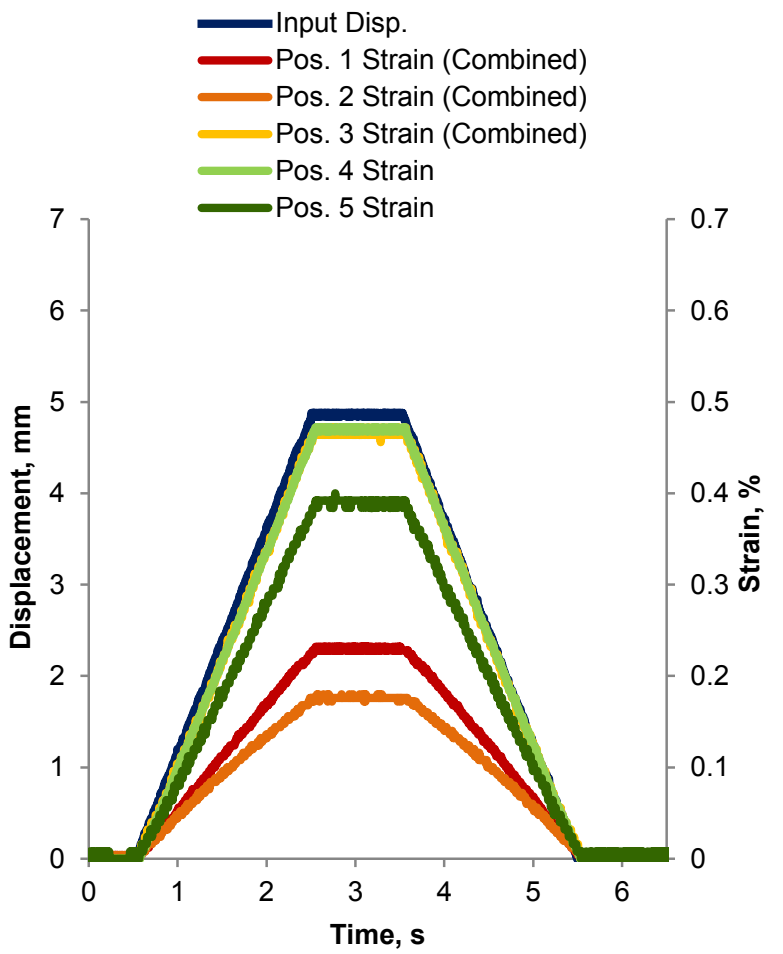

b)

Figure 16. Time-based strain measurements with input displacement for a) the $1 \mathrm{~mm}$ skin and b) $2 \mathrm{~mm}$ skin. 




Figure 17. Comparison of maximum strains obtained via finite element modelling and experimental measurements for both skins at the various locations.

\section{Conclusion}

A design chain featuring a flexible skin and a compliant and monolithic internal substructure was presented in this work. A demonstrator based on the results of these design-through-optimization tools demonstrates the usefulness of such design aids. The demonstrator also shows functionality of the concept, and with the modifications to some aspects of the design chain, in particular the reduction of greyness in final topologies and the prevention of the appearance of hinge-like regions, the concept can be feasible for the final wind tunnel test model. Results following the wind tunnel tests will provide further insight into the feasibility of such technologies in real aircraft implementation.

\section{Acknowledgments}

The presented work is carried out as part of the EU FP7 Project NOVEMOR and the authors thank the European Commission for the research funding (Grant Agreement 285395). Srinivas Vasista is a recipient of an Alexander von Humboldt Postdoctoral Research Fellowship and is grateful for the financial support from the Alexander von Humboldt Foundation. The authors would also like to acknowledge and thank Franziska Machtans for her support with experimental testing and Prof. Krister Svanberg for providing the method of moving asymptotes MATLAB codes.

\section{References}

1 CORDIS, "FP7: Transport (including Aeronautics)" Available: http://cordis.europa.eu/fp7/transport/. Accessed: September 01, 2012.

2 Thill, C., Etches, J., Bond, I., Potter, K., and Weaver, P., "Morphing Skins," Aeronautical Journal, Vol. 112, No. 1129, 2008, pp. 117-139.

3 Vasista, S., Tong, L., and Wong, K. C., "Realization of Morphing Wings: A Multidisciplinary Challenge," Journal of Aircraft, Vol. 49, No. 1, 2012, pp. 11-28, DOI: 10.2514/1.C031060.

4 Kintscher, M., Wiedemann, M., Monner, H. P., Heintze, O., and Kühn, T., "Design of a Smart Leading Edge Device for Low Speed Wind Tunnel Tests in the European Project SADE," International Journal of Structural Integrity, Vol. 2, No. 4, 2011, pp. 383-405, DOI: 10.1108/17579861111183911. 
5 Vasista, S., Monner, H. P., De Gaspari, A., and Ricci, S., "Morphing Devices for a Wing and Wingtip Based on Compliant Structures," 4th EASN Association Workshop on Flight Physics and Aircraft Design, Aachen, Germany, 27-29 October 2014.

6 Rudenko, A., Monner, H. P., and Rose, M., "A Process Chain for Structural Optimization of a Smart Droop Nose for an Active Blown High Lift System," 22nd AIAA/ASME/AHS Adaptive Structures Conference, 2014, pp. 1-6, DOI: $10.2514 / 6.2014-1414$.

7 Lagarias, J. C., Reeds, J. A., Wright, M. H., and Wright, P. E., "Convergence Properties of the Nelder--Mead Simplex Method in Low Dimensions," SIAM Journal on Optimization, Vol. 9, No. 1, 1998, pp. 112-147, DOI: $10.1137 / \mathrm{S} 1052623496303470$.

8 Lu, K.-J., and Kota, S., "Design of Compliant Mechanisms for Morphing Structural Shapes," Journal of Intelligent Material Systems and Structures, Vol. 14, No. June, 2003, DOI: 10.1177/104538903035563.

9 De Gaspari, A., and Ricci, S., "A Two-Level Approach for the Optimal Design of Morphing Wings Based On Compliant Structures," Journal of Intelligent Material Systems and Structures, Vol. 22, No. 10, 2011, pp. 10911111, DOI: $10.1177 / 1045389 X 11409081$.

10 Kang, Z., and Tong, L., "Integrated Optimization of Material Layout and Control Voltage for Piezoelectric Laminated Plates," Journal of Intelligent Material Systems and Structures, Vol. 19, No. 8, 2007, pp. 889-904, DOI: $10.1177 / 1045389 X 07084527$.

11 Luo, Z., Luo, Q., Tong, L., Gao, W., and Song, C., "Shape Morphing of Laminated Composite Structures with Photostrictive Actuators via Topology Optimization," Composite Structures, Vol. 93, No. 2, 2011, pp. 406-418, DOI: 10.1016/j.compstruct.2010.09.001.

12 Santer, M., and Pellegrino, S., "Topological Optimization of Compliant Adaptive Wing Structure," AIAA Journal, Vol. 47, No. 3, 2009, pp. 523-534, DOI: 10.2514/1.36679.

13 Maute, K. K., and Reich, G. W., "Integrated Multidisciplinary Topology Optimization Approach to Adaptive Wing Design," 2012.

14 Pedersen, C. B. W., Buhl, T., and Sigmund, O., "Topology Synthesis of Large-Displacement Compliant Mechanisms," International Journal for Numerical Methods in Engineering, Vol. 50, No. 12, 2001, pp. 26832705, DOI: $10.1002 /$ nme.148.

15 Thuwis, G. A., "Stiffness and Layout Tailoring of a Morphing High-Lift System with Aeroelastic Loads," PhD Thesis, TU Delft, 2012.

16 Bendsøe, M. P., and Sigmund, O., Topology Optimization - Theory, Methods and Applications, BerlinHeidelberg: Springer-Verlag, 2003.

17 Svanberg, K., "The Method of Moving Asymptotes - a New Method for Structural Optimization," International Journal for Numerical Methods in Engineering, Vol. 24, No. 2, 1987, pp. 359-373, DOI: $10.1002 / \mathrm{nme} .1620240207$.

18 Svanberg, K., "Some Modelling Aspects for the Matlab Implementation of MMA" Available: https://people.kth.se/ krille/mmamatlab.pdf. Accessed: December 01, 2014. 